\title{
Investigations on the effect of shape of traction separation law on the results of cohesive zone model
}

\author{
Rehan.M.Siddiqui ${ }^{1}$, Dattatray.N.Jadhav ${ }^{2}$, Nilesh.R.Raykar ${ }^{3}$ \\ ${ }^{1}$ Postgraduate student, Department of Mechanical engineering Sardar Patel College of \\ Engineering,Mumbai,India. \\ ${ }^{2}$ Associate professor, Department of Mechanical engineering Sardar Patel College of Engineering, Mumbai, \\ India. \\ ${ }^{3}$ Associate professor (Adhoc), Department of Mechanical engineering Sardar Patel College of Engineering, \\ Mumbai, India.
}

\begin{abstract}
The present study deals with the simulation of stable crack growth within a centre cracked plate made up of type 304 stainless steel material. Numerical crack propagation analyses have been performed considering elastic-plastic material behaviour under pure mode-I loading using $2 D$ cohesive zone modelling (CZM) with different traction separation laws (TSL). The study reports that the cohesive energy does not remain same for different TSL.There is a difference of 20-40\% in cohesive energy for different laws if employed to the same material.
\end{abstract}

Keywords: Cohesive Zone Model, Fracture, Node Release Technique, Traction Separation laws.

\section{Introduction}

The prediction of crack growth is at the heart of damage tolerant discipline. Failure in materials exhibits in three stages viz. crack initiation, stable crack growth and instability. Damage tolerant aims at monitoring the crack growth until the crack is likely to become unstable. After the crack initiation there is certain duration of time for which stable crack growth occurs. During this phase the component may be repaired or replaced, hence the study of stable crack growth becomes important. In very ductile materials such as type 304 stainless steel, it is well known that a sudden unstable crack extension hardly occurs even after the crack initiation. In these types of materials, the resistance to crack growth is also so large that the design of the structures becomes conservative if the crack initiation criterion is adopted as the failure condition of structures in the assessment of the structural integrity [1]. It is known, however, that even in such materials unstable fracture occurs after some amount of stable crack growth when the loading system has some amount of compliance [2,3]. In order to study such phenomenon, the analysis of stable crack growth is necessary and the finite element method is considered to be useful for this purpose.

Finite element analysis can be used to predict crack initiation and crack growth up to instability and beyond. The analysis can be based on continuum damage mechanics approach (e.g., Needleman and Tvergaard, 1987) and XFEM (e.g., Sukumar et al., 2000; Xiao and Karihaloo, 2006). To study crack growth there are approaches based on node release technique (e.g. G.Yagawa et al., 1984), crack tip opening displacement/angle (CTOD/CTOA) (e.g. D.N.Jadhav et al., 2009), J-resistance curve approach (J-R curve) (e.g., Xudong Qian et al., 2013), and the cohesive zone model (CZM) (e.g. D.N.Jadhav et al., 2009).

The cohesive zone model (CZM), based on the approach developed by Dugdale [9] and Barenblatt [10], has found increasing interest and applications in the past 20 years, because of its robustness, its limited set of parameters and broad range of possible applications. The CZM is a phenomenological model, it incorporates the mechanical processes near the crack tip that occur on crack propagation. Unlike other crack propagation models of finite element method (FEM) it does not require calculation of stress singularity at the crack tip. This simplifies the crack growth calculations. Within the cohesive zone, the damage and failure of a structure is modelled along a predefined crack path, by a damage-free bulk material and special interface elements called as cohesive elements in which the material separation takes place. The CZM does not represent any physical material, but describes the cohesive forces which occur when cohesive elements are pulled apart. The damage behaviour of the material within these elements is described by the so-called traction-separation law (TSL), which relates the magnitude of cohesive forces with the amount of separation. The law has two parameters, 
namely the maximum traction sustainable by the element, $\mathrm{T}_{0}$, and a maximum opening separation $\delta_{0}$, at which the element totally fails. Beside the two parameters $T_{0}$ and $\delta_{0}$, a third quantity can be defined as the energy dissipated by the cohesive element at total failure $\Gamma_{0}$. The cohesive energy represents the amount of energy supplied to the material during the period of crack initiation to the end of crack extension. The cohesive energy is related to the energy release rate in fracture mechanics [12-14]. Studies related to the cohesive law parameters including the variation of cohesive force with crack opening displacement have been reported by many investigators (e.g., Scheider and Brocks, 2003a,b). Yuan et al. [15] studied variations of the cohesive energy in crack initiation and subsequent crack propagation. Scheider et al [11] has shown that the transferability of the parameters from one TSL to another is not satisfied in general. This paper investigates the effect of the shape of TSL on the accuracy of predicting crack propagation within type 304 stainless steel by employing three shapes of TSL: trapezoidal, exponential and linear. Effect of mesh size onEffect of mesh size on the crack propagation analysis is also discussed.

\section{Simulation On Centre Cracked Plate}

Crack propagation is simulated on a centre cracked plate using node release technique and CZM with different TSL shapes.

\subsection{Specimen geometry and material}

The test specimen considered for the present analysis is a centre cracked plate of type 304 stainless steel as shown in Fig.1 [1]

The mechanical properties of type 304 stainless steel [1] are: young's Modulus E $=185.1 \mathrm{GPa}$, Yield stress $\sigma y=$ 225.4Mpa, Poisson's ratio $\mu=0.3$ for the strain hardening property the experimental data shown the Fig. 2 is used

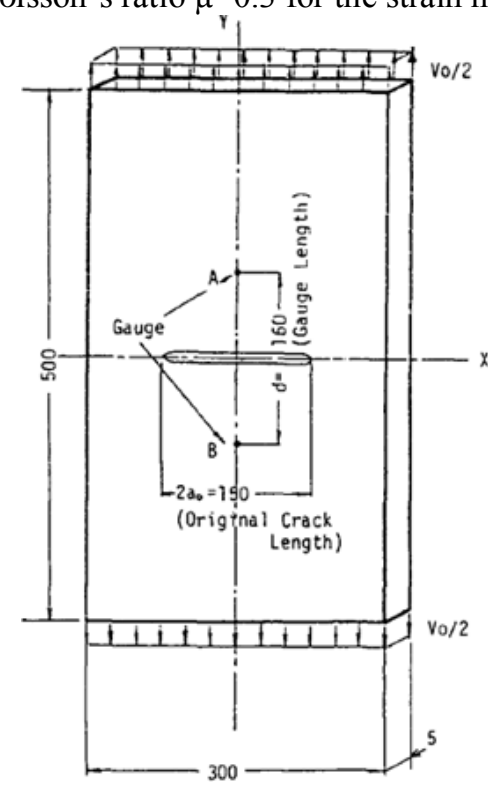

Figure.1.Center cracked plate analyzed (dimensions in $\mathrm{mm}$ ). [1]

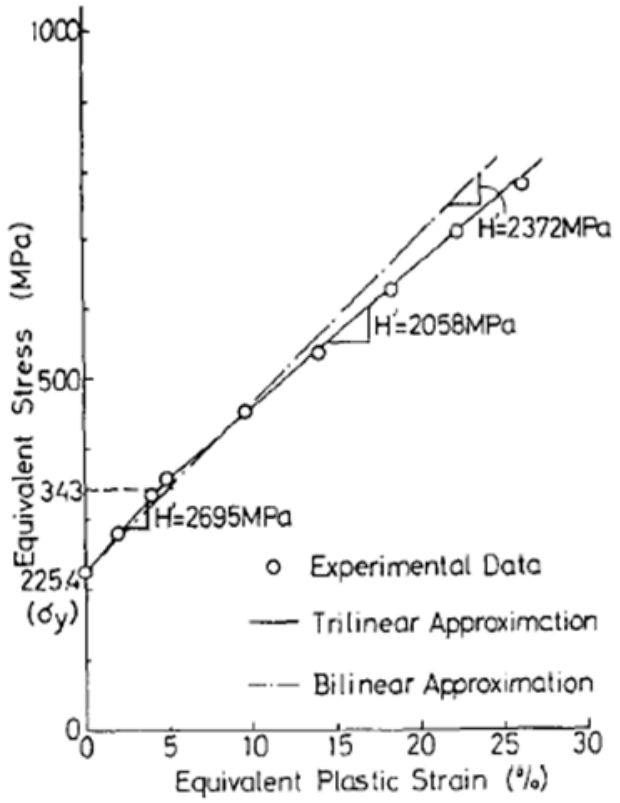

Figure.2. Equivalent Stress Vs Equivalent Plastic Strain.[1]

\subsection{Criterion for Crack extension (Node Release Technique)}

The criterion used for crack extension during node release technique is the experimental relation between the gauge point displacement and crack extension as shown in Fig.3. The gauge points are located at a distance of $160 \mathrm{~mm}$ along the centre line of the specimen as shown in Fig.1 


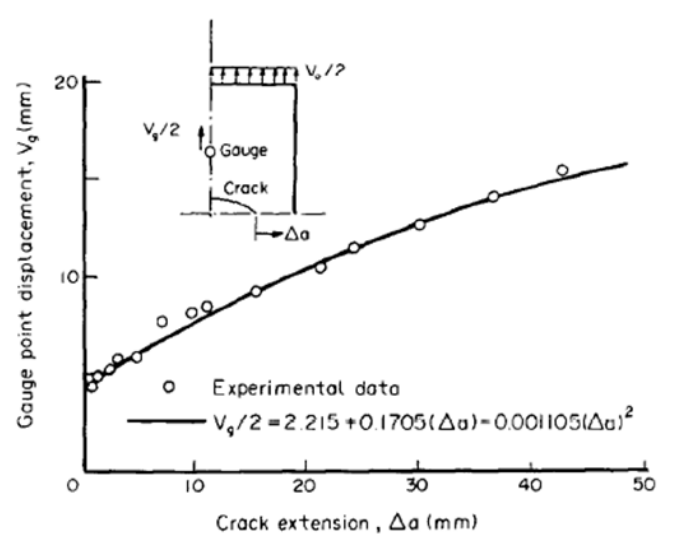

Fig.3 Gauge point displacement Vs crack extension [1]

\subsection{FE simulation}

In the present study to take advantage of symmetry, only right half part of the specimen was modelled. The cracked ligament was modelled with a layer of cohesive elements of negligible thickness. Since the thickness of specimen is $5 \mathrm{~mm}$ plain stress elements were employed in the analysis. The upper edge of the specimen was applied with uniform displacement, the lower edge was kept fixed and the left edge was given symmetric boundary condition. Multiple combinations of traction laws and variations in element sizes from $0.25 \mathrm{~mm}$ to $0.5 \mathrm{~mm}$ were checked. Element size of $0.5 \mathrm{~mm}$ was considered. Fig. 4 shows a finite element mesh with an element size of $0.5 \mathrm{~mm}$ along the crack front, there are 12615 second order quadrilateral elements and 150 cohesive elements.

For the node release technique a separate mesh with an element length of $1 \mathrm{~mm}$ along the crack front was used, only right quarter part of the plate was modelled. The boundary conditions were identical to that employed in CZM. The plate was meshed with second order quadrilateral shell elements. In this case, at the instance of meeting criteria of sec.2.2 the crack is extended discontinuously by one element length by releasing the restraint of the crack tip node and giving it several load increments to cancel its nodal force component in the direction perpendicular to the crack surface. The mid-side node is released at the same time to avoid incompatibility on the unopened part of the element side.
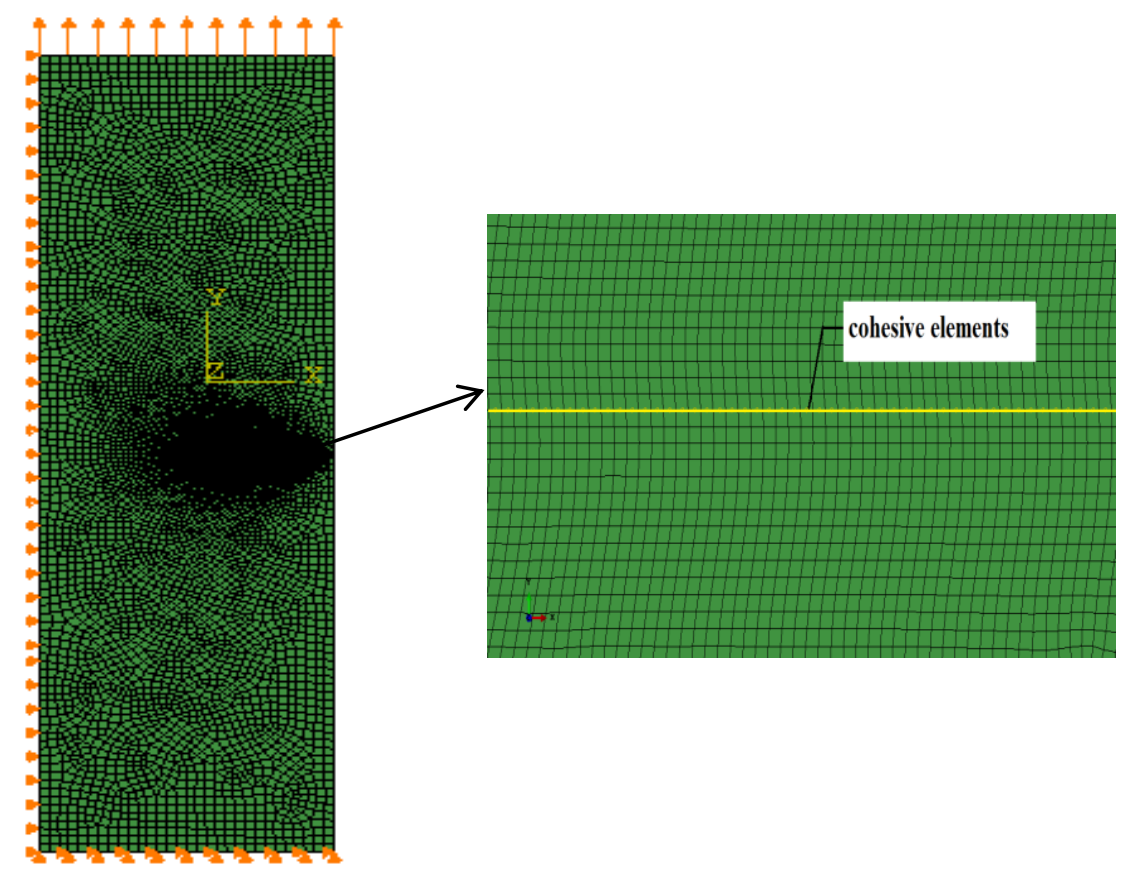

(a) (b)

Figure.4 (a) Finite element mesh along with boundary conditions. (b) cohesive elements along the crack front 


\subsection{Traction-separation law and cohesive parameters}

The selection of appropriate form of the TSL has been attracted much interest in the past. For the ductile materials researchers [11] suggest use of exponential or constant variation (Trapezoidal) shape of TSL. In the present study the linear exponential and constant traction law are been compared. Fig.5 shows the form of traction separation laws
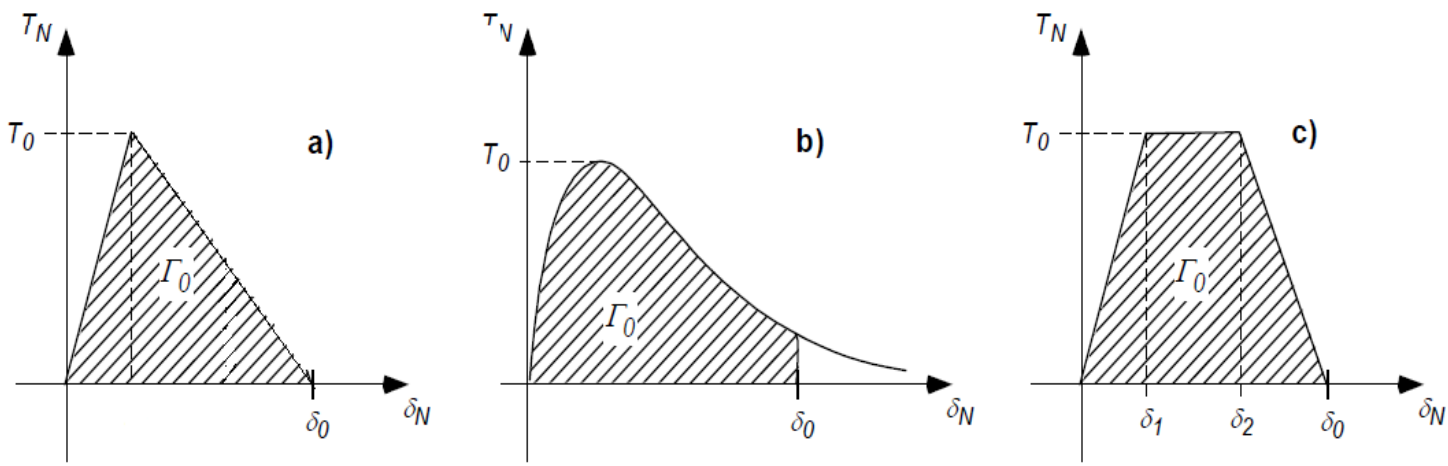

Figure.5.Traction-separation laws: a) Linear function, b) Exponential function,c.) Trapezoidal function In the exponential TSL Fig.5b dependence of the traction on the separation is given by [11]

$T(\delta)=T_{0} z \frac{\delta}{\delta_{0}} e\left(1-z\left(\delta / \delta_{0}\right)\right) \ldots \ldots$ where $z=16 e / 9$

Hence the corresponding fracture energy is given by $\Gamma_{0}=\frac{9}{16} T_{0} \delta_{0}$

The Trapezoidal traction-separation law is more versatile TSL, in which a region can be defined, where the traction in the cohesive element is constant.

This has been achieved by the use of two additional parameters, $\delta_{1}$ and $\delta_{2}$, leading to the following formulation for the function $\mathrm{T}(\delta)[11]$

$$
T=T_{0}\left(\begin{array}{l}
2\left(\frac{\delta}{\delta 1}\right)-\left(\frac{\delta}{\delta 1}\right)^{2} \ldots \ldots . . \delta<\delta_{1} \\
1 \ldots \ldots \ldots \ldots \ldots \ldots \ldots \ldots \delta_{1}<\delta<\delta_{2} \\
2\left(\frac{\delta-\delta_{2}}{\delta_{0}-\delta_{2}}\right)^{3}-\left(\frac{\delta-\delta_{2}}{\delta_{0}-\delta_{2}}\right)^{3}+1 \ldots \ldots . . \delta_{2}<\delta<\delta_{0}
\end{array}\right.
$$

For crack growth simulation, the two parameters, i.e., maximum normal traction/cohesive strength $\mathrm{T}_{0}$ and as the maximum/critical normal separation $\delta_{0}$, are required to be given as input. Based on experimental stress-strain data [1] stress corresponding to final fracture was assumed to be 3 times yield stress and this was taken as the cohesive strength $\left(\mathrm{T}_{0}\right)$. J at crack initiation i.e. $1.04 \mathrm{MN} / \mathrm{m}$ as calculated by G.Yagawa et al., 1984 [1] was taken as cohesive energy $\Gamma_{0}$. Substituting value of $\Gamma_{0}$ in Eq. (2), $\delta_{0}$ value is obtained, which is used as input in FE analysis. Similar procedure was adopted for other laws also. Correct value of cohesive energy was obtained iteratively so as to match the experimental crack growth data.

\section{Results And Discussions}

As the load increases, the stress in the cohesive element also increases as this stress reaches the cohesive strength the degradation of cohesive elements start., for the crack extension the separation and stresses are calculated in every increment for all cohesive elements at the integration points according to the traction separation law. When the critical separation is reached the element fails at this point, the contribution of the integration point to the stiffness vanishes and the point obtains the status failed, once the element fails it gets deleted thereby forming new crack edges. Fig.6.shows the cohesive elements just at the start of crack extension. 


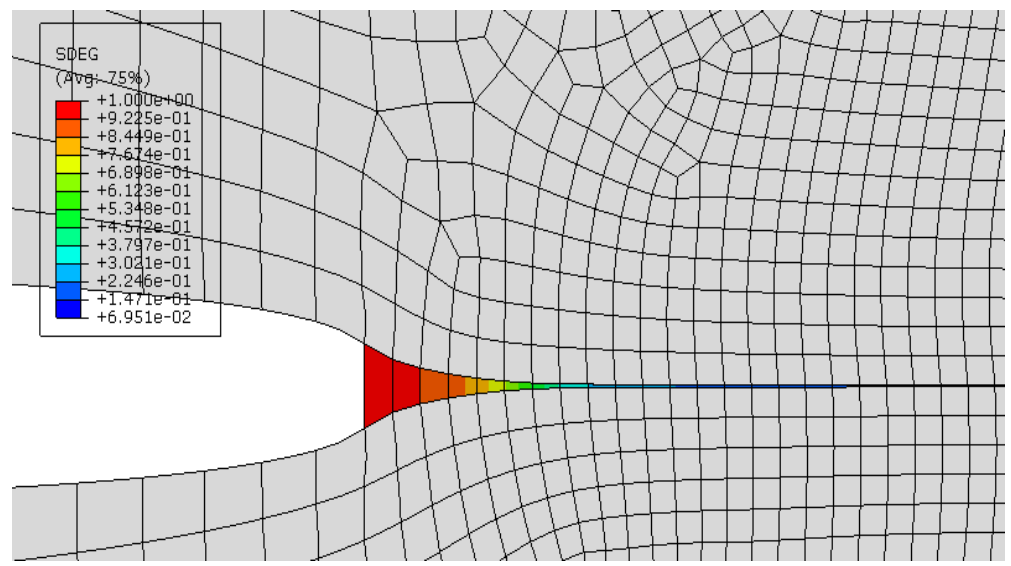

Figure.6. Cohesive elements at the start of crack extension (trapezoidal TSL)

\subsection{Gauge point displacement comparison}

Fig.7. shows the predicted variation of the gauge point displacement and crack extension, along with the experimental data [1]. The values obtained from the trapezoidal law are in good agreement with the experimental values, while the values of linear law are in good agreement with the experimental values up to $16 \mathrm{~mm}$ crack extension but the displacement of gauge point lowers after $16 \mathrm{~mm}$ of crack extension, while the exponential law shows a higher gauge point displacement at crack initiation and converges with the experimental values at $12 \mathrm{~mm}$ of crack extension.

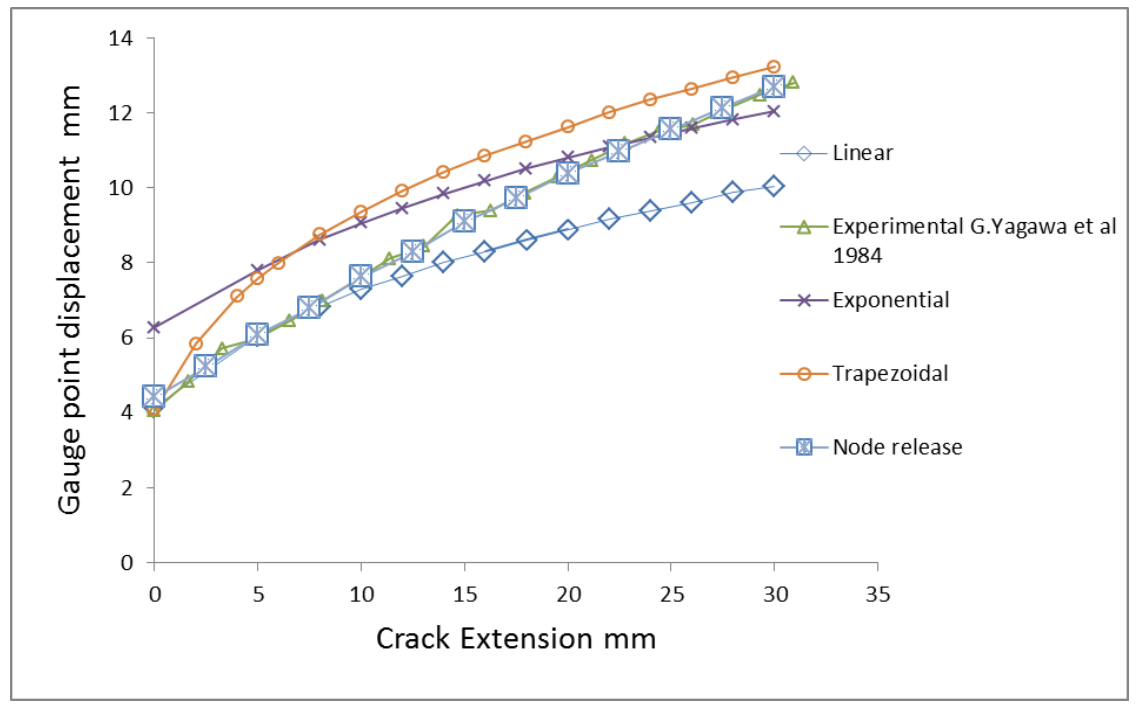

Figure.7. Gauge point displacement vs crack extension

\subsection{Load comparison}

$\mathrm{T}$ he variation of the gauge point displacement and the load during crack extension is shown in Fig.8. The load at crack initiation and maximum load in the experiment is around $295 \mathrm{KN}$ and $300 \mathrm{KN}$ respectively. The load values predicted by node release method and those by CZM with trapezoidal TSL agree well with each other. The CZM with linear and exponential TSL show a more decrease in load after crack initiation. The maximum load in case of node release technique is $259 \mathrm{KN}$ while in case of CZM with trapezoidal law is $270 \mathrm{KN}$. The CZM results gives $10 \%$ lower values of the maximum load which is better than the node release result with $13.33 \%$ error.

\subsection{Influence of Mesh size}

To check for the influence of the mesh size on accuracy of results an element size of $0.25 \mathrm{~mm}$ was also considered for the trapezoidal case. The results of the gauge point displacement vs crack extension and load vs crack extension are shown in Fig.9 and Fig.10 respectively.From figures it is seen that both the mesh size $0.25 \mathrm{~mm}$ and $0.5 \mathrm{~mm}$ seem to converge with each other, hence to save computational resources, element size of $0.5 \mathrm{~mm}$ is considered. 


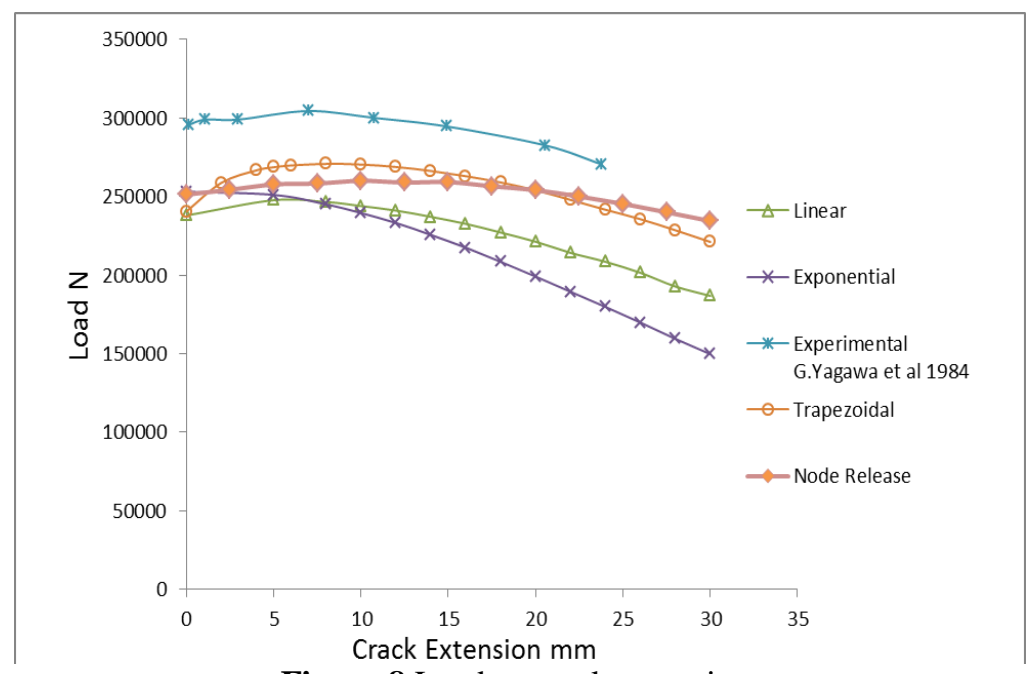

Figure.8 Load vs crack extension

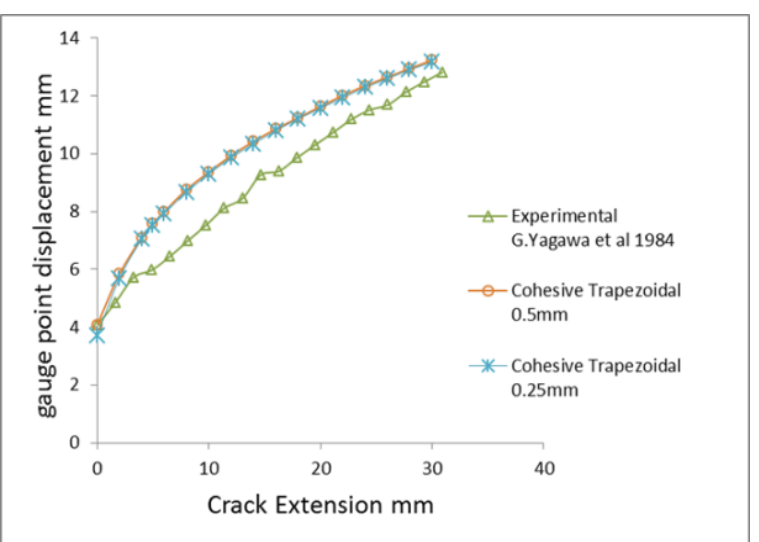

Figure.9 Gauge Point Displacement vs crack extension trapezoidal TSL

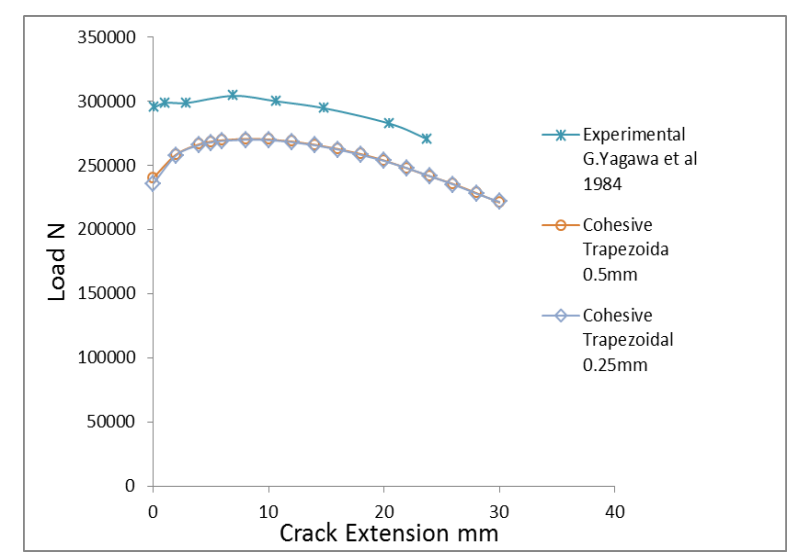

Figure.10 Load vs crack extension trapezoidal TSL

\subsection{Comparison of Cohesive Energy employed by different TSL shapes}

The cohesive energy $\Gamma_{0}$ for different TSL shapes which gave better match between the experimental values and numerical values of variation of gauge point displacement and crack extension are given in Table.1. These cohesive energies may be assumed to represent the J-Integral at crack initiation $\left(\mathrm{J}_{\mathrm{i}}\right)$. The $\mathrm{Ji}$ in case of node release technique was calculated using the inbuilt function of ABAQUS.

Table.1 Cohesive Energies for different TSL shapes

\begin{tabular}{|l|l|}
\hline Type of Law/Method & Cohesive energy $\left(\mathbf{J}_{\mathbf{i}}\right) \mathrm{MN} / \mathrm{m}$ \\
\hline Node Release & 1.04 \\
\hline Linear Cohesive law & 0.76 \\
\hline Exponential Cohesive law & 1.265 \\
\hline Trapezoidal Cohesive law & 1.022 \\
\hline
\end{tabular}

The cohesive energy obtained for each TSL shape is distinct. The cohesive energy in case of trapezoidal TSL is near to the $\mathrm{J}_{\mathrm{i}}$ of node release technique. Further the gauge point displacement curve and load vs crack extension curves for trapezoidal case show better agreement with the experimental values.

\section{Conclusions}

In the present study crack growth was simulated on a centre cracked plate using Node release technique and CZM employing linear, exponential and trapezoidal TSL. The study reports that the shape of TSL affects the results of the crack simulation using CZM. For a specific material there is specific TSL with suitable parameters which suits the crack simulation properly. In this case for type 304 Stainless steel material the trapezoidal TSL is better for crack simulation. For the trapezoidal case both the gauge point displacement curve and load vs crack extension curve are in better agreement with the curve obtained from experimental values. Also the cohesive energy needed to match with the experimental data is different for different TSL shapes 
employed to the same material. There is a difference of $20-40 \%$ in cohesive energy for different laws if employed to the same material.

\section{References}

[1]. G. Yagawa, Y. Takahashi and K. Kashima, Elastic-Plastic analysis on stable crack growth for center cracked plate: A benchmark study, Engineering Fracture Mechanics Vol. 19. No. 4, pp. 155-169. 1984.

[2]. G. Yagawa, Y. Takahashi and Y. Ando, Theoretical and experimental study on unstable fracture for type 304 stainless steel plates with a soft tensile testing machine. Engineering Fracture Mech. M(5), 721-731, 1982.

[3]. G. M. Wilkowski A. Zaboor and M. F. Kanninen, A plastic fracture mechanics prediction of fracture instability in a circumferentially cracked pipe in bending-Part 2: Verification on a type 304 stainless steel pipe. ASME Journal of.Pressure Vessel Tech. 103(4) 359-365 1981.

[4]. Needleman, A., Tvergaard, V, An analysis of ductile rupture modes at a crack tip. J. Mech. Phys. Solids 35, 151-183, 1987.

[5]. Sukumar, N.Moes, N.Moran, B.Belytschko, Extended finite element for three dimensional crack modelling Int. J. Numeical. Method ins Eng. 48, 1549-1570,2000.

[6]. Xiao, Q.Z., Karihaloo, B.L., Improving the accuracy of XFEM crack tip fields using higher order quadrature and statically admissible stress recovery. Int. J. Numerical. Methods in Eng. 66, 1378-1410,2006.

[7]. D.N. Jadhav, S.K. Maiti, Characterization of stable crack growth through AISI 4340 steel using cohesive zone modeling and CTOD/CTOA criterion.Nuclear Engineering and Design 240,713-721,2010.

[8]. Xudong Qian,Yang Zhang, Yoo Sang Choo, A load-deformation formulation with fracture representation based on the J-R curve for tubular joints, Engineering Failure Analysis, Volume 33, 347-366,2013.

[9]. Dugdale DS, Yielding of steel sheets containing slits. J Mech Phys Solids;8:100-4, 1960.

[10]. Barenblatt, G.I, The mathematical theory of equilibrium cracks in brittle fracture. Adv. Appl. Mech. 7, 55-129,1962

[11]. Scheider, I., Brocks, W., 2003a, The effect of the traction separation law on the results of cohesive zone crack propagation analyses. Key Engg.Materials., 313-318,2003

[12]. Scheider, I., Brocks, W., 2003b. Simulation of cup-cone fracture using cohesive model. Eng. Fract. Mech. 70, 1943-1961,2003

[13]. Needleman A. An analysis of decohesion along an imperfect interface. Int Journal of Fracture ;42:21-40,1990.

[14]. Tvergaard V, Hutchinson JW. The relation between crack growth resistance and fracture process parameters in elastic-plastic solids. J Mech Phys Solids;40:1377-97,1992.

[15]. Huang Yuan, Xiao Li, Effects of the cohesive law on ductile crack propagation simulation by using cohesive zone models.Engineering Fracture Mechanics 126 1-11, 2014 